\title{
Localized High Expression of Type-A Response Regulator and Cytokinin Oxidase/dehydrogenase Genes in Relation to Forchlorfenuron-induced Changes in Flower Morphology in Torenia fournieri Lind.
}

\author{
Tomoya Niki ${ }^{1,2}$, Taximaimaiti Mahesumu ${ }^{3}$, Tomoko Niki ${ }^{1}$ and Takaaki Nishijima ${ }^{1,2 *}$ \\ ${ }^{1}$ NARO Institute of Floricultural Science, Tsukuba 305-8519, Japan \\ ${ }^{2}$ Graduate School of Life and Environmental Sciences, Tsukuba University, Tsukuba 305-8577, Japan \\ ${ }^{3} Y i l i$ Teachers' College, Yining 835000, Xinjiang Uygur Autonomous Region, China
}

\begin{abstract}
Several morphological changes in flowers can be induced in torenia (Torenia fournieri L.) by applying forchlorfenuron (CPPU) to flower buds. We investigated the temporal and spatial distributions of the cytokinin response in CPPU-treated flower buds, which is indicated by type-A response regulator (RR) and cytokinin oxidase (CKX) gene expression. The quantitative real-time PCR analysis showed that the expression of both T. fournieri RRI (TfRRI) and TfCKX5 was induced from 1 day after CPPU treatment in the sepal, petal, stamen, and pistil, and maintained at a high level until 5 days after the treatment, when the earliest morphological changes due to CPPU treatment were observed. In situ hybridization analysis showed weak expression of both genes in the stamen and pistil through all floral stages of non-treated flower buds. However, when CPPU was applied at the sepal development stage, expression of both genes was strongly induced in the abaxial side of the stamen primordia, which is the site of initiation of the wide paracorolla. When CPPU was applied during the early stage of corolla development, high expression of those genes was observed in the stamen, basal, and middle part of the petal, which is the site of initiation of the narrow paracorolla. Those high levels of expression became more strongly localized to the paracorolla initiation site once the paracorolla primordia formed. When CPPU was applied during the middle corolla development stage, strong expression of those genes was detected in the middle to apical parts of the petal, which is the site of changes in the distribution pattern of the vascular bundles and the resultant serrated margin. These results suggest that long-term elevation of cytokinin signaling caused by CPPU treatment induces changes in flower morphology, and the paracorolla and serrated margin of the petal are induced by localized high levels of cytokinin signaling at the site of those morphological changes within flower buds.
\end{abstract}

Key Words: cytokinin signal localization, floral stage, flower morphology, paracorolla, spatial distribution.

\section{Introduction}

One of the most important traits for a floricultural plant is impressive flower morphologies such as double flowers, flowers with a paracorolla, and large flowers. These decorative flowers have an impact on commercial values; thus, improvement of flower morphology is a major objective of breeding programs. In general, mutation breeding has been employed to obtain such

Received; August 6, 2012. Accepted; October 30, 2012.

This work was supported by the Japan Society for the Promotion of Science (a Grant-in-Aid KAKENHI No. 17658018 and 22580045). Parts of this paper were presented at the 2007 autumn and 2009 spring meetings of the Japanese Society for Horticultural Science.

* Corresponding author (E-mail: takaaki@affrc.go.jp). flowers; however, the desired stable mutants can usually be obtained only at a very low frequency (Nishijima, 2007). Thus, elucidation of the molecular mechanisms responsible for decorative flowers will help to develop an efficient breeding method based on molecular techniques such as DNA markers and genetic transformation.

It has been shown that cytokinin is involved in the regulation of flower morphology. In Arabidopsis, the number of petals, stamens, and pistils can be increased by application of $N^{6}$-benzylaminopurine (BA) to flower buds (Lindsay et al., 2006; Venglat and Sawhney, 1996). These increases in floral organ numbers are due to the enlarged floral meristem that is caused by cytokininmediated regulation of the genes involved in meristematic activity and organ differentiation, i.e., WUSCHEL 
and CLAVATA (Bartrina et al., 2011; Clark et al., 1993; Lindsay et al., 2006; Rupp et al., 1999). In Petunia hybrida, the application of forchlorfenuron (CPPU), an inhibitor of cytokinin oxidase/dehydrogenase (CKX), to flower buds leads to corolla enlargement through an increase in cell numbers (Nishijima et al., 2006). Furthermore, genes involved in cytokinin biosynthesis and early signaling pathways are regulated by alleles of the Grandiflora gene, which is responsible for the largeflowered phenotype in petunia (Nishijima et al., 2011a, b). Application of CPPU to the flower buds of torenia induces some morphological changes including an increase in petal numbers, a serrated petal margin, and formation of a paracorolla (Nishijima and Shima, 2006). Interestingly, these flower morphologies depend on the floral stage at CPPU treatment. When CPPU is applied at the sepal development stage (Stage 3), petal-like wide paracorollas are induced and narrow paracorollas are induced by CPPU treatment at the early corolla development stage (Stage 5). On the other hand, serrated petals are induced by CPPU treatment at the middle corolla development stage (Stage 6). These results indicate that these flower morphologies could be induced by the specific spatial distribution of cytokinin concentration in flower buds, depending on the floral stage. Of these flower morphologies, paracorollas are formed only in a few plant species including Narcissus, Asclepias, Antirrhinum majus, and Passiflora, contributing to the outstanding and unique appearance of the flowers of these species. Thus, it would be possible to produce flowers with a totally new appearance if it is possible to induce paracorollas in other floricultural plants.

It has been shown that the morphological differences observed in CPPU-induced torenia paracorollas are regulated by the expression pattern of floral homeotic genes (Niki et al., 2012). In the wide paracorollas, which have the same morphological and anatomical characteristics as petals, class A and B genes are expressed mostly as they are in the initiation site, i.e., the basal part of the petal. On the other hand, in the narrow paracorollas having either petal-like or stamen-like characteristics, class B genes are expressed mostly as they are in the initiation site, i.e., the middle part of the petal. Thus, it would be possible to regulate paracorolla morphology by altering the expression of floral homeotic genes.

Since CPPU inhibits CKX activity, CPPU-induced morphological changes in the torenia flower should be caused by elevation of endogenous cytokinin concentration (Bilyeu et al., 2001). Different floral morphologies are induced depending on the floral stage at CPPU treatment, as mentioned above. Thus, we speculated that the spatial distribution of high cytokinin concentrations in CPPU-treated flower buds differ depending on the floral stage. Although it is difficult to investigate cytokinin distribution in young flower buds, we can determine the distribution of the cytokinin response by monitoring the expression level of the genes involved in cytokinin biosynthesis and signal transduction. The early cytokinin signal transduction pathway consists of a two-component signaling system (Mizuno, 2005; Müller, 2011). Binding of a biologically active cytokinin to the receptor histidine protein kinase induces His-Asp phosphorelay to histidine phosphotransfer proteins (HPt). The phosphorylated HPt phosphorylates and activates type-B response regulators (RRs), which act as transcription factors and induce the expression of type-A RRs. Type-A RRs act as a repressor of cytokinin signaling, which causes a negative feedback loop to cytokinin signaling. The expression of type-A $R R \mathrm{~s}$ is rapidly and strongly induced in response to cytokinin, and thus, the expression level reflects the extent of the cytokinin signal (Brandstatter and Kieber, 1998; D'Agostino et al., 2000; Nishijima et al., 2011b; Taniguchi et al., 1998). In addition, CKX is involved in negative feedback regulation of cytokinin biosynthesis, because $C K X \mathrm{~s}$ expression is induced by cytokinin (Brugière et al., 2003; Kiba et al., 2005; Nishijima et al., 2011a). Thus, the expression of type-A $R R \mathrm{~s}$ and $C K X \mathrm{~s}$ may be used as indices of cytokinin signaling.

In this study, we investigated the spatial and temporal expression patterns of type-A $R R \mathrm{~s}$ and $C K X \mathrm{~s}$ in CPPUtreated flower buds to clarify the localization of cytokinin signaling when the paracorollas and serrated petals are induced. Furthermore, based on these results, a strategy to induce particular flower morphologies by regulating cytokinin signaling in flower buds is discussed.

\section{Materials and Methods}

\section{Plant materials}

Seeds of Torenia fournieri, 'Dwarf White' (Sakata Seed Co., Kanagawa, Japan), were germinated in horticultural soil (Metro-Mix 350; Sun Gro Horticulture Canada Ltd., British Columbia, Canada), and the seedlings were transplanted to another horticultural soil (Kureha-Engei-Baido; Kureha Chemical Industry Co., Ltd., Tokyo, Japan) in plastic pots and then grown in an incubator kept at $25^{\circ} \mathrm{C} / 20^{\circ} \mathrm{C}$ (day/night) under illumination from fluorescent lamps of $180 \mu \mathrm{mol} \cdot \mathrm{m}^{-2} \cdot \mathrm{s}^{-1} \mathrm{PPFD}$ (12 h light/12 h dark).

\section{$C P P U$ and BA treatment}

The CPPU (Sigma-Aldrich Japan Co., Ltd., Tokyo, Japan) and BA (Wako Pure Chemical Industries Ltd., Osaka, Japan) solution was prepared as described by Nishijima and Shima (2006). We applied $8 \mu \mathrm{L}$ of $3 \mu \mathrm{M}$ CPPU or $8 \mu \mathrm{L}$ of $100 \mu \mathrm{M}$ BA solution to the apex of an inflorescence. To ensure the induction of the morphological changes, flower buds longer than $10 \mathrm{~mm}$ were removed before the treatment.

cDNA cloning of type- $A R R$ and CKX genes and phylogenetic analyses

The methods for total RNA isolation from young flower buds using an RNeasy Plant Mini Kit (Qiagen 
Sciences, Germantown, MD, USA) and RNase-Free DNase Set (Qiagen), and cDNA synthesis using a CapFishing Full-length cDNA Premix Kit (Seegene, Seoul, Korea) were described previously (Niki et al., 2012). Degenerate primers of type-A $R R$ and $C K X$ genes were designed using highly conserved regions of each gene, and partial cDNAs were amplified using the following degenerate primers: forward 5'-CAYGTIYT IGCIGTIGAYGA-3' and reverse 5'-YTSIGCICCYT CYTCIARRCA-3' for type-A $R R$ genes, and some combination of forward 5'-GTIKCIGCIMGIGGICAIGG ICA-3', 5'-TGGACIGAYTAYYTIYAYYTIACIGTIGG3', 5'-GGIGGIYTIGGICARTTYGGIRTIATHAC-3', 5'TGGGAIGTICCICAYCCITGGYTIAA-3'，5'-CCIGTIT CITGGACIGAYTAYTTRTA-3' and reverse 5'-TGICCI GGIGMIARIAKIGYYHKIGGRTC-3'， 5'-TTIARCCA IGGRTGIGGIACITCCCA-3'，5'-GTDATIAYICCRAA YTGICCIARICCICC-3', 5'-TGICCIGGIGAYAAIAGII TYTTIGGRTCRAA-3' for $C K X$ genes. Based on the sequences of the PCR products, gene-specific primers were designed, and $5^{\prime}$ and $3^{\prime}$ RACE were performed using the CapFishing Full-length cDNA Premix Kit (Seegene). To isolate full-length cDNAs for each gene, PCRs were performed with KOD Plus DNA polymerase (Toyobo, Osaka, Japan) using the following primers: forward 5'-TTACCTCTCATCACTGTAACGCA-3' and reverse 5'-AATGAAACAACTGACTTGGAAATTC-3' for T. fournieri RRI (TfRRI), forward 5'-TACTATGAT TCTGTAGGTTGGCGT-3' and reverse 5'-GCAGCG CACCTCAATTATAAG-3' for TfRR2, forward 5'-TTC CCCTCCTCATCTTACACC-3' and reverse 5'-TTGCC ATAAAGCGTCGAAAT-3' for $T f C K X 1$, forward 5'-CA CAAAATCACGCACTGACACA-3' and reverse 5'-CA GAATAATTAACAATTACCATTGCG-3' for TfCKX2, forward 5'-CTTTCCTTCCTACGGTCAAATC-3' and reverse 5'-TGAAGCAAAGGCAGGACTAAC-3' for TfCKX3, forward 5'-ACTTTCAAGAATCTCGACAG CA-3' and reverse 5'-AATTCGATAGTAAAAGCGC ATA-3' for TfCKX4, and forward 5'-ACCACACT AAAATCATACTCTCCTC-3' and reverse 5'-CCACT AATATTAAAAATGTAAACTCCAC-3' for TfCKX5. Each PCR fragment was cloned into a pGEM-T Easy vector (Promega, Madison, WI, USA) and the nucleotide sequence was analyzed with a BigDye Terminator v3.1 Cycle Sequencing Kit and an ABI PRISM 3100 Genetic Analyzer (Applied Biosystems, Foster City, WI, USA). Accession numbers of the cloned cDNAs are listed in the legend for Figure 1.

For phylogenetic analyses, the full length of each amino acid sequence of type-A RR or CKX was used, respectively. Predicted amino acid sequences of the cloned type-A $R R$ or $C K X$ genes from torenia were compared with those of Arabidopsis by using CLUSTAL W (http://clustalw.ddbj.nig.ac.jp/top-j.html). The phylogenetic trees were constructed using the neighbor-joining method and were drawn with NJplot (http://pbil.univlyon1.fr/software/njplot.html).
Quantitative real-time PCR analysis

Total RNA was isolated separately from the sepals, petals, stamens, and pistils of flower buds after CPPU or BA treatment and those of non-treated controls using the same procedure used for cDNA cloning. cDNA was synthesized using a Transcriptor First Strand cDNA Synthesis Kit (Roche, Mannheim, Germany). Genespecific primers for $T f R R$ and $T f C K X$ genes and the actin gene (TfACT3; AB330989), which was used as an internal control, were designed for the 3 '-terminal regions of the open reading frame and the 3 '-untranslated regions of each gene. Primer sequences and the lengths of PCR products used for the quantitative real-time PCR (qPCR) reactions were as follows: forward 5'-AGATTATTAGT TGTTCTCCTCTGT-3' and reverse 5'-CTTGGAAAT TCAACCACATCA-3' for TfRR1 (155 bp), forward 5'GCTGCAATGTTGAAGAACATG-3' and reverse 5'CAGCGCACCTCAATTATAAG-3' for TfRR2 (155 bp), forward 5'-CCCATATCAGTTTTGTGACACA-3' and reverse 5'-CATACTTACAGTTGTTGAGGAGGA-3' for TfCKXI (124 bp), forward 5'-CCGTTGATTAATCCTA GTG-3' and reverse 5'-AGAGAGACAATCACGATAC ATC-3' for TfCKX2 (158 bp), forward 5'-TCAAGAAAT TGGAAGAAGGCC-3' and reverse 5'-CCAATATATA ATTCATTTCCCCACT-3' for TfCKX3 (169 bp), forward 5'-CCAAAGACTTGGAACAACAGTG-3' and reverse 5'-GATTGCTCTACATCTGAGAGACC-3' for TfCKX4 (146 bp), forward 5'-AGAAAGTTGAAGTTCGATCC CG-3' and reverse 5'-TTACATTCCACAGACCACA ACTG-3' for TfCKX5 (117 bp), and forward 5'-TG CAGTAAAGTGTATTGTGGAAG-3' and reverse 5'-GG AACTATCTGGGTAGGATC-3' for TfACT3 (145 bp). Expression of the genes was quantified using SYBR Premix Ex Taq (Takara Bio, Shiga, Japan) and qPCR (LightCycler; Roche). PCR reactions were performed with an initial denaturation step of $10 \mathrm{~s}$ at $95^{\circ} \mathrm{C}$, followed by 50 cycles of $5 \mathrm{~s}$ at $95^{\circ} \mathrm{C}, 10 \mathrm{~s}$ at $60^{\circ} \mathrm{C}$, and $5-7 \mathrm{~s}$ at $72^{\circ} \mathrm{C}$. Fluorescence was measured at the end of the extension phase at $73^{\circ} \mathrm{C}$ for $T f C K X 1$ and $T f C K X 2$, at $74^{\circ} \mathrm{C}$ for $T f C K X 3$, at $75^{\circ} \mathrm{C}$ for $T f R R 2$, at $76^{\circ} \mathrm{C}$ for $T f R R 1$, at $77^{\circ} \mathrm{C}$ for $T f A C T 3$, and at $78^{\circ} \mathrm{C}$ for $T f C K X 4$ and $T f C K X 5$, to avoid detecting non-specific PCR products. The raw data were analyzed with LightCycler software version 3.5 (Roche). The plasmids harboring full-length cDNA of TfRR or TfCKX genes or a partial cDNA fragment of TfACT3 were used to obtain the standard curves. The ratio of the expression of each gene to that of TfACT3 was calculated. Expression analyses were conducted independently in triplicate.

\section{In situ hybridization}

Flower buds at 3 or 7 days after CPPU treatment and those of non-treated controls were used for in situ hybridization. The flower buds were fixed in FAA $(50 \%$ $(\mathrm{v} / \mathrm{v})$ ethanol, $10 \%(\mathrm{v} / \mathrm{v})$ formaldehyde, $5 \%(\mathrm{v} / \mathrm{v})$ acetic acid) and embedded in paraffin as described by Niki et al. (2012), and the tissues were sectioned ( $8 \mu \mathrm{m}$ thick) 
with a rotary microtome (RM2145; Leica, Nussloch, Germany). The in situ hybridization was performed as described by Niki et al. (2012), except the hybridization temperature and the method of RNA probe preparation were modified. The sections were dewaxed and rehydrated as described in the morphological analysis section. After washing with PBS $(0.1 \mathrm{M} \mathrm{NaCl}, 10 \mathrm{mM}$ $\mathrm{NaH}_{2} \mathrm{PO}_{4}, 10 \mathrm{mM} \mathrm{Na}_{2} \mathrm{HPO}_{4}, \mathrm{pH}$ 7.4), the sections were treated with $1 \mu \mathrm{g} \cdot \mathrm{mL}^{-1}$ proteinase $\mathrm{K}$ (Roche) in PBS at $37^{\circ} \mathrm{C}$ for $30 \mathrm{~min}$ and the reaction was stopped by washing with PBS containing $0.2 \%$ glycine. After washing with PBS, the sections were acetylated in $0.1 \mathrm{M}$ triethanolamine $\mathrm{HCl}(\mathrm{pH} 8.0)$ and $0.25 \%$ acetic anhydride for 20 min. After incubation with 1\% Triton X-100 in PBS and further washing with PBS, the sections were prehybridized at room temperature for $2 \mathrm{~h}$ in a hybridization buffer containing $50 \%$ formamide, $4 \times$ saline-sodium citrate (SSC), 1× Denhardt's solution, $1 \mathrm{mg} \cdot \mathrm{mL}^{-1}$ Escherichia coli tRNA, and $0.5 \mathrm{mg} \cdot \mathrm{mL}^{-1}$ salmon sperm DNA, and then hybridized with gene-specific digoxigenin (DIG)-labeled antisense or sense (control) RNA probes for TfRRI or TfCKX5. The RNA probes were prepared from PCR fragments with the T7 and SP6 promoter sequence of each gene with a DIG RNA Labeling Kit (Roche). The PCR fragments were generated with the following primers: forward 5'-GAATAATACGACTCA CTATAGGGTCAGAGATTTCGTTATCAAAGGC-3' and reverse 5'-TGCATTTAGGTGACACTATAGAAATG AAACAACTGACTTGGAAATTC-3' for TfRR 1 $(180 \mathrm{bp})$, and forward 5'-GAATAATACGACTCACT ATAGGGCTGCTGTTGTACCAGATGAAGAC-3' and reverse 5'-TGCATTTAGGTGACACTATAGAACCAC TAATATTAAAAATGTAAACTCCAC-3' for TfCKX5 (154 bp). DIG-labeled RNAs were synthesized by T7 (for sense probe) or SP6 (for anti-sense probe) RNA polymerases with a DIG RNA Labeling Kit (Roche). After purification by ethanol precipitation, the RNAs were used as probes. Hybridization was performed with each probe at a concentration of $800 \mathrm{ng} \cdot \mathrm{mL}^{-1}$ in hybridization buffer at $62^{\circ} \mathrm{C}$ overnight. After hybridization, the sections were washed in $0.2 \times \mathrm{SSC}$ at $65^{\circ} \mathrm{C}$ for $2 \mathrm{~h}$, and then in NT buffer $(0.15 \mathrm{M} \mathrm{NaCl}, 0.1 \mathrm{M}$ Tris$\mathrm{HCl}, \mathrm{pH}$ 7.5) at room temperature. After treatment with $1 \%$ blocking reagent (Roche) for $1 \mathrm{~h}$, the sections were incubated with diluted $(1: 500)$ anti-DIG-AP (Roche) for $1 \mathrm{~h}$. Chemical staining was performed with NBT/ BCIP solution (Roche). Hybridization analyses were conducted independently in triplicate using independent CPPU-treated flower buds.

\section{Results and Discussion}

cDNA cloning and phylogenetic analyses of torenia type$A R R$ and $C K X$ genes

We isolated two type-A $R R$ (TfRRI and 2) and five $C K X(T f C K X 1,2,3,4$, and 5) genes from torenia cDNA. The deduced amino acid sequences of the isolated genes were highly homologous to Arabidopsis type-A RRs
(ARRs) and CKXs, respectively. In addition to the conserved amino acid sequence of Asp that needs HisAsp phosphorelay, the short C-terminal sequence without a GARP domain suggested that TfRR1 and 2 are typeA RRs (D'Agostino et al., 2000; Mizuno, 2005). In amino acid sequences of C-terminal region, TfRR1 had a characteristic with Asp, Ser, and Thr rich sequences as those of ARR7 and 15 (D'Agostino et al., 2000). All TfCKXs had a conserved FAD binding domain and cytokinin binding domain (Schmülling et al., 2003). The phylogenetic analysis showed that TfRRI belonged to the same clade as $A R R 5,6,7$, and 15 which are known to be induced by cytokinin and repressed specifically in the shoot apex by WUSCHEL (D'Agostino et al., 2000; Leibfried et al., 2005), and TfRR2 belonged to the same clade as $A R R 16$ and 17 (Fig. 1A). Similarly, TfCKXI, 2, 3, 4, and 5 were homologous to $\operatorname{AtCKX6}, \operatorname{AtCKX}$, $A t C K X 7, A t C K X 1$, and $A t C K X 3$, respectively (Fig. 1B).

\section{Induction of TfRR and TfCKX genes in floral organs by $C P P U$ treatment}

The expression of both type-A $R R$ and $C K X$ genes is known to be induced by cytokinin; however, the extent of the induction differs greatly among the members of the gene family (D'Agostino et al., 2000; Kiba et al., 2005). To investigate the responsiveness of each gene to cytokinin, we analyzed the expression pattern of $T f R R \mathrm{~s}$ and $T f C K X \mathrm{~s}$ in each floral organ 2 days after CPPU treatment.

The results of qPCR analyses showed low expression of TfRRI in all floral organs of non-treated flower buds; however, CPPU treatment increased the expression approximately 4-fold in the stamen and pistil, approximately 8 -fold in the sepal, and approximately
(A)

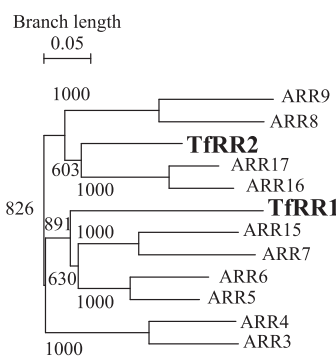

(B)

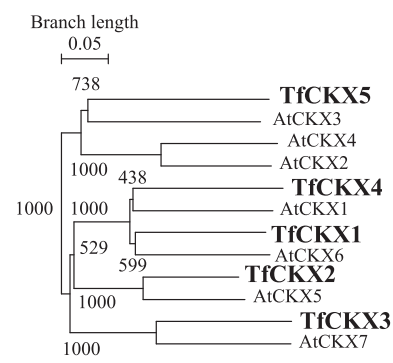

Fig. 1. Phylogenetic tree of type-A RR and CKX in torenia and Arabidopsis. Bootstrap values from 1000 replicates are indicated near the branching points. Accession numbers were as follows: torenia type-A RR genes, TfRR1, AB740033; TfRR2, AB740034; Arabidopsis type-A RR genes, ARR3, At1g59940; ARR4, At1g10470; ARR5, At3g48100; ARR6, At5g62920; ARR7, Atlg19050; ARR8, At2g41310; ARR9, At3g57040; ARR15, At1g74890; ARR16, At2g40670; ARR17, At3g56380; torenia CKX genes, TfCKX1, AB740035; TfCKX2, AB740036; TfCKX3, AB740037; TfCKX4, AB740038; TfCKX5, AB740039; Arabidopsis CKX genes, AtCKX1, At2g41510; AtCKX2, At2g19500; AtCKX3, At5g56970; AtCKX4, At4g29740; AtCKX5, Atlg75450; AtCKX6, At3g63440; AtCKX7, At5g21482. 
10 -fold in the petal, respectively (Fig. 2). In contrast, $T f R R 2$ expression was very low and induction of the expression by CPPU was unclear (Fig. 2). Of all $T f C K X \mathrm{~s}$, $T f C K X 5$ was more highly expressed than the other $T f C K X \mathrm{~s}$ in all floral organs of non-treated flower buds, suggesting that $T f C K X 5$ mainly functions in the flower of torenia (Fig. 2). Furthermore, the expression of $T f C K X 5$ was clearly induced by CPPU treatment in all floral organs, whereas no induction of $T f C K X 1, T f C K X 2$, or $T f C K X 4$ was observed in any floral organ. Although

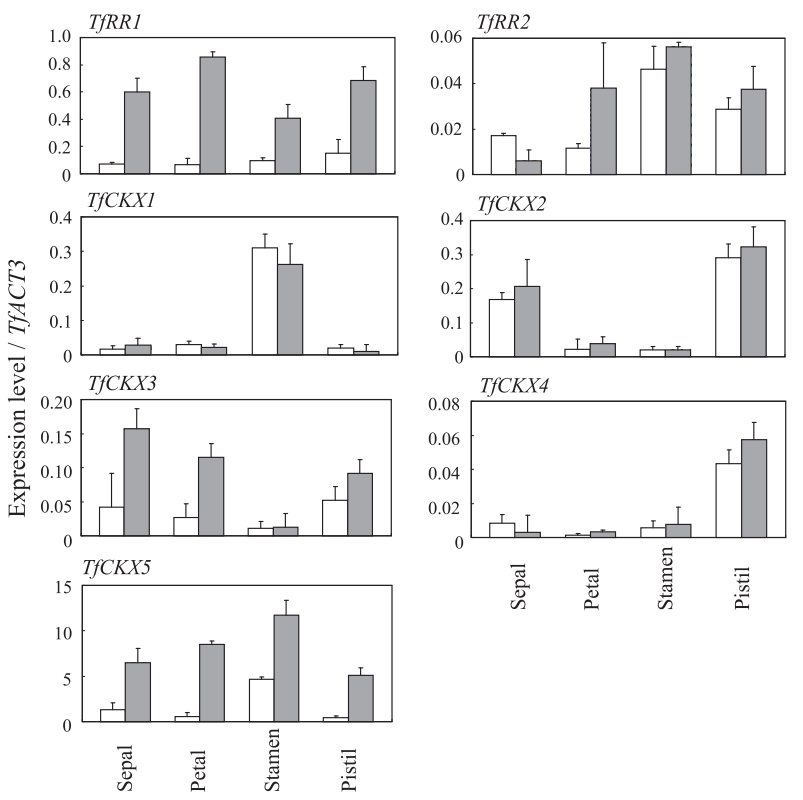

Fig. 2. Quantitative real-time PCR analyses of TfRR and TfCKX in floral organs 2 days after CPPU treatment. The expression levels are shown as a relative value against TfACT3 as an internal standard. Open and gray columns indicate non-treated and CPPU-treated floral organs, respectively. Vertical bars indicate $\mathrm{SE}(\mathrm{n}=3)$.

(A) TfRRI

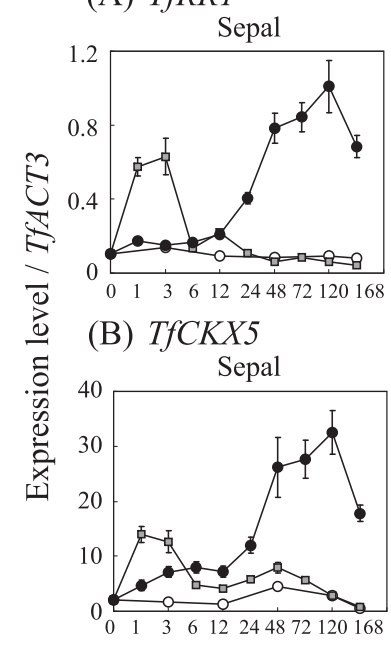

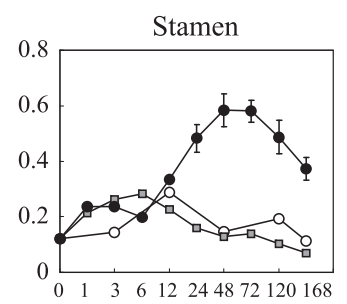
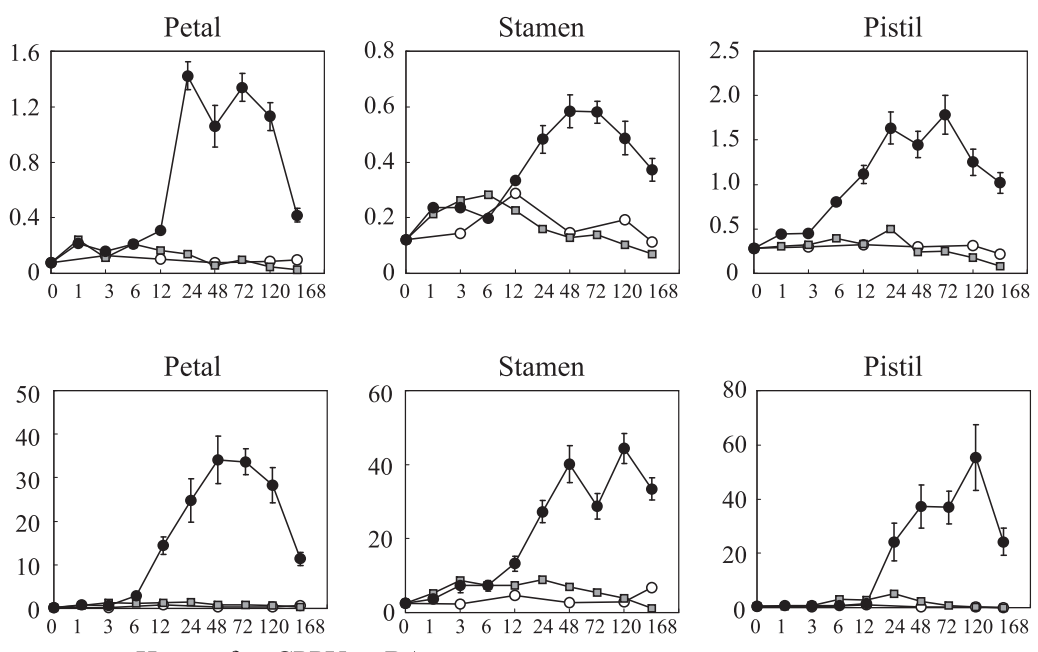

$T f C K X 3$ was induced by CPPU in the sepal and petal, the expression was much lower than that of TfCKX5 (Fig. 2). These results indicate that the expression of $T f R R 1$ and $T f C K X 5$ is clearly induced in all floral organs by CPPU treatment. Thus, these genes have a high probability to function as indicators of cytokinin signaling.

The expression of TfRRl was induced from 1 day after CPPU treatment in all floral organs (Fig. 3A). The expression was maintained at a high level until 5 days after the treatment, and decreased 9 days after the treatment (Fig. 3A). Approximately the same expression pattern was observed in $T f C K X 5$; expression was induced from $12 \mathrm{~h}$ after the treatment in the petal, 1 day in the stamen and pistil, and 2 days in the sepal and was maintained at a high level until 5 days and decreased 9 days after the treatment (Fig. 3B). These results clearly suggest that cytokinin signaling is greatly increased by CPPU treatment and the high level continues until 7 days after the treatment, when the earliest CPPU-induced morphological changes in flower buds were observed (Niki et al., 2012; Nishijima and Shima, 2006). This continuous high level of cytokinin signaling may be the cause of these changes in floral morphology.

In contrast, BA treatment increased the expression of both TfRR 1 and TfCKX5 $1-3 \mathrm{~h}$ after the treatment only in the sepal, and the level decreased to the same level observed in the control $6 \mathrm{~h}$ after the treatment (Fig. 3A, $\mathrm{B})$. The expression level of those genes in the petal, stamen, and pistil was not changed by BA treatment (Fig. 3A, B). It has been shown that no morphological change in flowers is observed by BA treatment in torenia (Nishijima and Shima, 2006). Those observations coincide with our results that cytokinin signaling was not elevated by BA treatment in torenia (Fig. 3A, B). Flower buds of torenia are completely enclosed by the sepals after initiation of the stamen and pistil (Stage 4).

Fig. 3. Quantitative real-time PCR analyses of TfRR1 and TfCKX5 in CPPU or BA-treated floral organs. The expression levels are shown as a relative value against TfACT3 as an internal standard. Open circles, closed circles, and gray squares indicate non-treated control, CPPU or BA-treated flower buds, respectively. Vertical bars indicate SE $(n=3)$. 
The sepals of young flower buds have densely developed trichomes. The chemical solution seemingly cannot penetrate the sepals to reach the inside of the flower buds; thus, elevation of cytokinin signaling by BA treatment may have been limited to the sepals (Fig. 3A, B). In contrast, CPPU may have been translocated to other floral organs after being absorbed by the sepals and thus, its effects were long-lasting in flower buds. Furthermore, different chemical structures between BA and CPPU might cause the different extent of translocation (Mok and Mok, 2001). BA, which has an isoprenoid side chain, may be degraded markedly by elevated CKX activity induced by BA treatment. In contrast, CPPU, a diphenylurea compound, is not degraded by CKX (Bilyeu et al., 2001).

\section{Spatial expression analyses of TfRR and TfCKX genes in CPPU-treated flower buds}

The results of in situ hybridization showed that in non-treated flower buds of Stage 3 and 4 weak signals of both TfRRI and TfCKX 5 were detected in the stamen and pistil primordia (Fig. 4A-a, b, d, f, g, i). When CPPU was applied to flower buds at Stage 3 , strong expression of both TfRRI and TfCKX5 was detected in the stamen and pistil primordia 3 days after the treatment and the area of expression extended to the adaxial side of the sepals (Fig. 4A-c, h). In particular, TfCKX5 was strongly expressed in the abaxial side of the stamen primordia, which is the site of initiation of the wide paracorolla (Fig. 4A-h). At 7 days after CPPU treatment, when the primordia of the wide paracorollas were initiated, strong expression of both TfRRI and TfCKX 5 was observed not only in the stamen and pistil primordia, but also in the primordia of the wide paracorollas (Fig. 4A-e, j).

Low expression of both TfRRI and TfCKX5 was detected in the stamen and pistil primordia at Stage 5 of non-treated flower buds, and the expression was limited to the anther and ovule at Stage 7 (Fig. 4B-a, b, $\mathrm{d}, \mathrm{f}, \mathrm{g}, \mathrm{i}$ ). When CPPU was applied to flower buds at Stage 5 , both genes were strongly expressed in the stamen and pistil 3 days after the treatment. TfRRI was expressed in the entire petal, whereas TfCKX5 was strongly expressed in the middle and basal parts of the petal; the middle part of the petal is the site of initiation of the narrow paracorolla (Fig. 4B-c, h). In the flower buds 7 days after CPPU treatment, strong expression of both TfRRI and TfCKX5 was observed in the stamen and pistil; however, expression was limited to the anthers and ovule, as observed in non-treated flower buds (Fig. 4B-d, e, i, j). Furthermore, strong expression of both genes was observed in the middle to the apical part of the petal, whereas the expression level was low in the basal part of the petal (Fig. 4B-e, j).

These results indicate that cytokinin signaling is elevated by CPPU treatment at the paracorolla initiation site. When CPPU was applied to flower buds at Stage 3 , cytokinin signaling was elevated on the abaxial side of the stamen primordia, which is the site of initiation of the wide paracorolla, although high levels of cytokinin signaling were also observed in the petals (Figs. 4A-c, $\mathrm{e}, \mathrm{h}, \mathrm{j}$ and 5). When CPPU was applied to flower buds at Stage 5, cytokinin signaling was first elevated in the petal and stamen, and later the elevated cytokinin signaling was occurred in the middle part of the petal, which is the site of initiation of the narrow paracorolla (Figs. 4B-c, e, h, j and 5).

Morphologically, the paracorolla resembles the petals, and it originates from the stipule of the stamen in Narcissus, Asclepias, and A.majus, or from the receptacle in Passiflora (Troll, 1957; Yamaguchi et al., 2010). The wide paracorollas of torenia are formed at the abaxial and lateral side of the stamen primordia, indicating they apparently originate from the stipule of the stamen (Nishijima and Shima, 2006). The narrow paracorollas are also formed on the lateral side of the stamen; however, the primordia of the narrow paracorollas are relatively separate from the stamen, because they are formed on the border between the limb and tube of a rather developed petal. Since the basal part of the stamen is fused with the tube in torenia, the basal part of the narrow paracorolla may be fused with the petal tube. Thus, the narrow paracorolla is seemingly formed just at the lateral side of the stamen, similar to the wide paracorolla concerning its fusion to the tube. CPPU elevated cytokinin signaling at the abaxial side of the basal part of the stamen when the wide paracorollas were induced, and at the middle and basal part of the petal and basal part of the stamen when the narrow paracorollas were induced (Figs. 4A, B and 5). These results suggest that the high level of cytokinin signaling in CPPU-treated flower buds is localized to the site where the stipule of the stamen, i.e., the paracorolla, originates.

Localized auxin accumulation called 'localized auxin maxima' at the site of organ formation acts as a trigger of the organogenic process (Benková et al., 2003; Reinhardt et al., 2000). Cytokinins modulate the spatial distribution of auxin via regulation of auxin efflux, and it is evident that spatial distribution of distinct endogenous cytokinin levels is necessary to form localized auxin maxima (Pernisová et al., 2009), although the detailed relationship between the distribution pattern of endogenous cytokinin and that of auxin maxima is still unknown. In our experiment, CPPU caused localized strong cytokinin signaling in flower buds of torenia, which was not observed in untreated flower buds (Fig. 4). Thus, it is probable that ectopically formed auxin maxima was caused by CPPU treatment, which may have triggered to form an extra organ, i.e., the paracorolla formation.

When CPPU was applied to flower buds at Stage 6, which induced the serrated petal margin, both TfRRI and $T f C K X 5$ were strongly expressed in the anther, pistil, and middle to apical part of the petal 3 days after the 
(A)

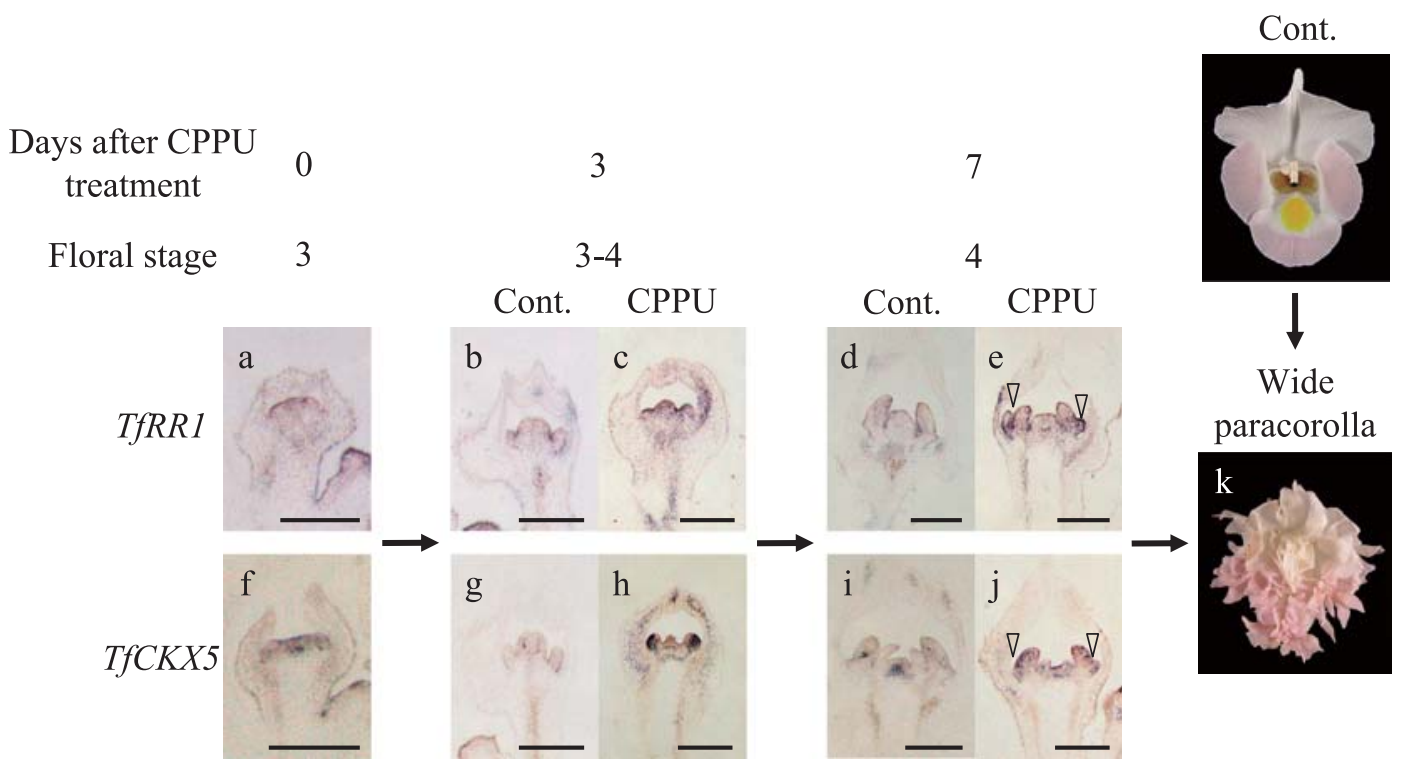

(B) Days after CPPU treatment

3

7

Floral stage 5

6

7

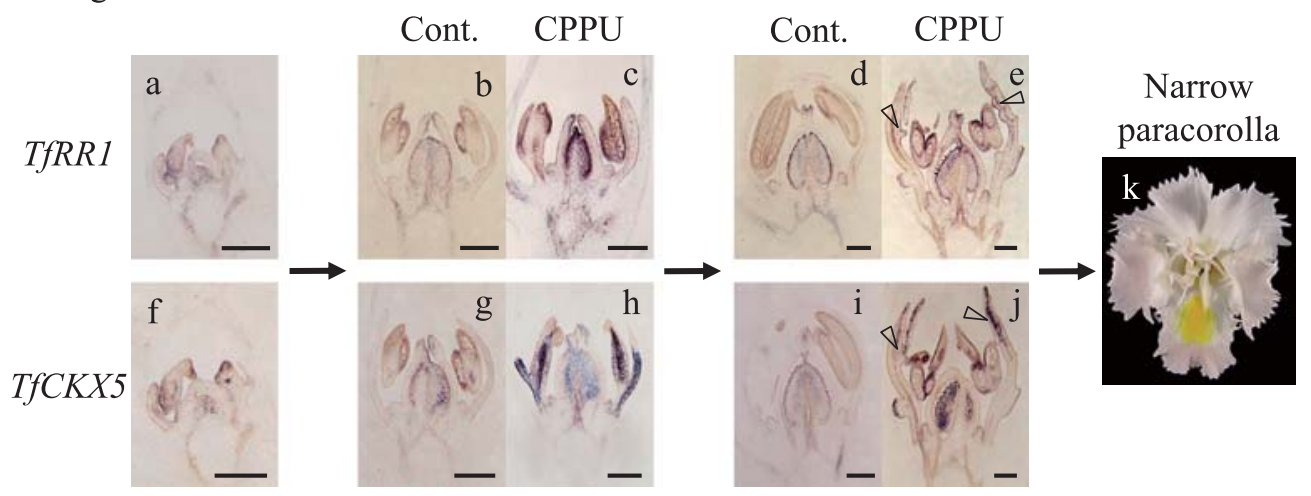

(C) Days after CPPU

treatment

3

7

Floral stage 6

7

7

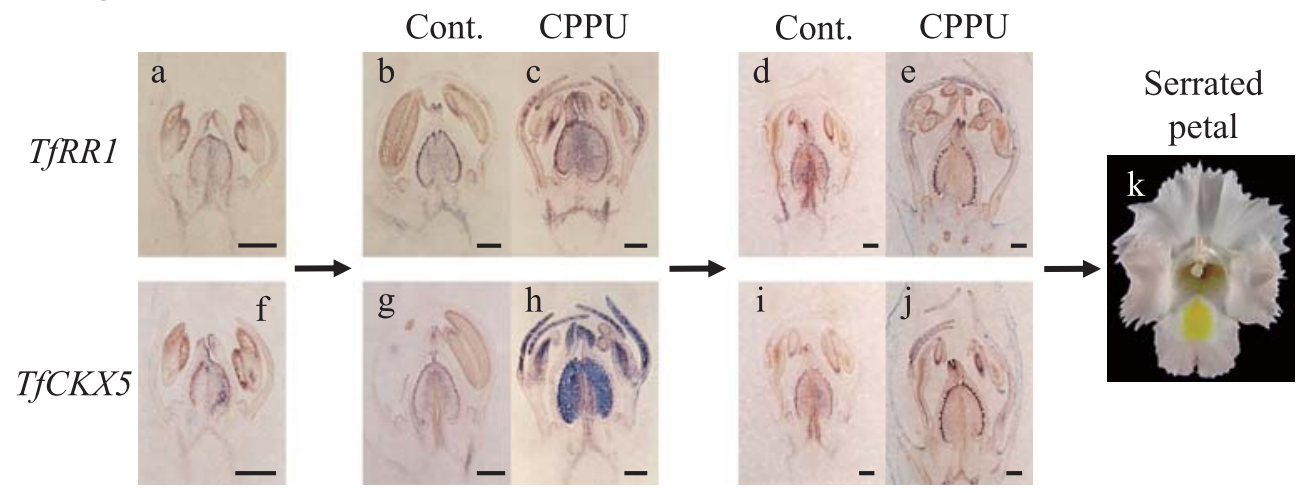

Fig. 4. In situ hybridization of TfRR1 and TfCKX5 in flower buds treated with CPPU. CPPU treatment induces formation of a wide paracorolla in (A), a narrow paracorolla in (B), and a serrated petal margin in (C). CPPU-treated flower buds were collected at 3 (c, h) or 7 days (e, j) after the treatment, whereas non-treated flower buds were collected at the corresponding stage (a, b, d, f, g, i). The representative data at each floral stage inducing each flower morphology are shown. Panel $\mathrm{k}$ shows flower morphology induced by CPPU treatment at each floral stage. Panel b, d, g, and i in (B) are the same as a, b, f, and g in (C), respectively, because each shows the same stage of non-treated flower buds. Floral stages were defined as described in Nishijima and Shima (2006). Triangles represent the initiation site of paracorollas. Scale bars $=200 \mu \mathrm{m}$. 


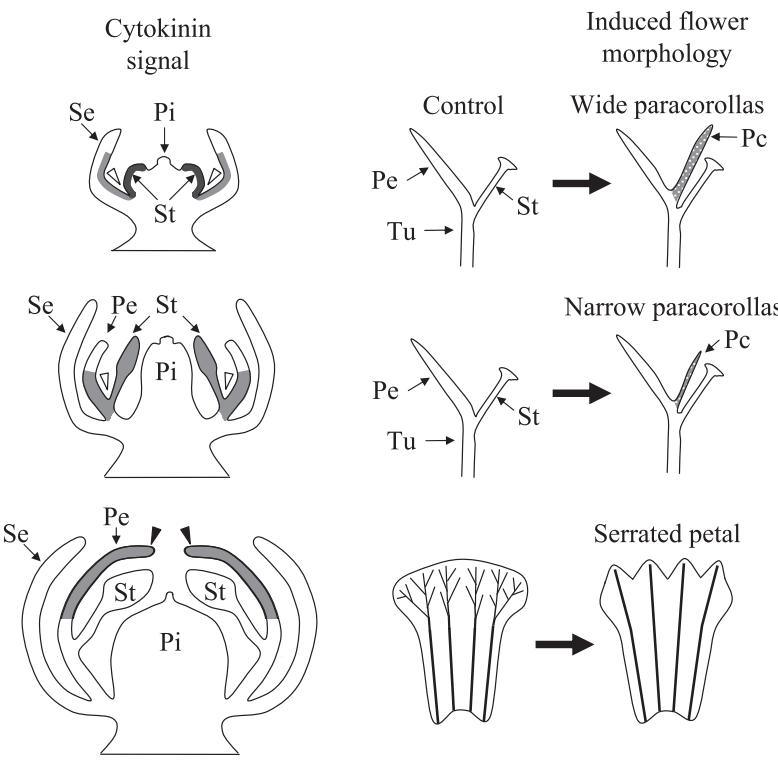

Fig. 5. Hypothetical model of distribution of cytokinin signaling in flower buds and CPPU-induced flower morphology in torenia. The floral organs with high levels of cytokinin signaling are shade gray, and paracorollas are meshed gray. Triangles represent the initiation site of paracorollas $(\triangle)$ and serrated margin $(\boldsymbol{\Delta})$. Pc, Paracorolla; Pe, Petal; Pi, Pistil; Se, Sepal; St, Stamen; Tu, Tube.

treatment, whereas weak expression of both genes was detected in the anther and ovule of non-treated flower buds (Fig. 4C-a, b, c, f, g, h). However, the expression of both genes in CPPU-treated flower buds was very low at the basal part of both the petal and stamen (Fig. 4C-c, h). The site of high expression of both genes 7 days after CPPU treatment was limited to the apical part of the petal, which corresponds to the limb (Fig. 4C$\mathrm{e}, \mathrm{j}$ ). In contrast to the spatial distribution of the high levels of cytokinin signaling observed when paracorolla induction occurs, high levels of cytokinin signaling were limited to the middle to apical part of the petal when the serrated petal was induced by CPPU (Figs. 4A-c, h, $\mathrm{B}-\mathrm{c}, \mathrm{h}, \mathrm{C}-\mathrm{c}, \mathrm{h}$, and 5). CPPU changes the arrangement of vascular bundles from an intensively branched arrangement to a non-branched parallel one in the whole limb, which causes the serrated petals (Nishijima and Shima, 2006). Therefore, the site of CPPU-induced high levels of cytokinin signaling, i.e., the whole limb, corresponds to the site of the morphological changes causing the serrated petal, i.e., changes in the spatial arrangement of vascular bundles (Figs. 4C and 5).

In summary, CPPU induces long-term elevation of cytokinin signaling in flower buds, which induces changes in flower morphology. Further, localization of high levels of cytokinin signaling to the site of morphological changes at specific floral stages induces formation of the paracorolla and serrated petals. Thus, it may be possible to induce the desired flower morphology by localizing cytokinins to a particular site of flower buds at an appropriate floral stage. That is, the wide paracorolla can be induced when cytokinin is accumulated in the abaxial side of stamen primordia at the sepal development stage, the narrow paracorolla can be induced when cytokinin is accumulated in the basal part of the stamen to the middle of the petal during the early corolla development stage, and serrated petals can be induced when cytokinin is accumulated in the limb at the middle corolla development stage.

\section{Acknowledgement}

We thank Mrs. Tomoko Kurobe for her technical assistance.

\section{Literature Cited}

Bartrina, I., E. Otto, M. Strnad, T. Werner and T. Schmülling. 2011. Cytokinin regulates the activity of reproductive meristems, flower organ size, ovule formation, and, thus, seed yield in Arabidopsis thaliana. Plant Cell 23: 69-80.

Benková, E., M. Michniewicz, M. Sauer, T. Teichmann, D. Seifertová, G. Jürgens and J. Friml. 2003. Local, effluxdependent auxin gradients as a common module for plant organ formation. Cell 15: 591-602.

Bilyeu, K. D., J. L. Cole, J. G. Laskey, W. R. Riekhof, T. J. Esparza, M. D. Kramer and R. O. Morris. 2001. Molecular and biochemical characterization of a cytokinin oxidase from maize. Plant Physiol. 125: 378-386.

Brandstatter, I. and J. J. Kieber. 1998. Two genes with similarity to bacterial response regulators are rapidly and specifically induced by cytokinin in Arabidopsis. Plant Cell 10: 10091019.

Brugière, N., S. Jiao, S. Hantke, C. Zinselmeier, J. A. Roessler, X. Niu, R. J. Jones and J. E. Habben. 2003. Cytokinin oxidase gene expression in maize is localized to the vasculature, and is induced by cytokinins, abscisic acid, and abiotic stress. Plant Physiol. 132: 1228-1240.

Clark, S. E., M. P. Running and E. M. Meyerowitz. 1993. CLAVATA1, a regulator of meristem and flower development in Arabidopsis. Development 119: 397-418.

D'Agostino, I. B., J. Deruère and J. J. Kieber. 2000. Characterization of the response of the Arabidopsis response regulator gene family to cytokinin. Plant Physiol. 124: 1706-1717.

Kiba, T., T. Naitou, N. Koizumi, T. Yamashino, H. Sakakibara and T. Mizuno. 2005. Combinatorial microarray analysis revealing Arabidopsis genes implicates in cytokinin responses through the His $\rightarrow$ Asp phosphorelay circuitry. Plant Cell Physiol. 46: 339-355.

Leibfried, A., J. P. C. To, W. Busch, S. Stehling, A. Kehle, M. Demar, J. J. Kieber and J. U. Lohmann. 2005. WUSCHEL controls meristem function by direct regulation of cytokinininducible response regulators. Nature 438: 1172-1175.

Lindsay, D. L., V. K. Sawhney and P. C. Bonham-Smith. 2006. Cytokinin-induced changes in CLAVATA1 and WUSCHEL expression temporary coincide with altered floral development in Arabidopsis. Plant Sci. 170: 1111-1117.

Mizuno, T. 2005. Two-component phosphorelay signal transduction systems in plants: From hormone responses to circadian rhythms. Biosci. Biotechnol. Biochem. 69: 2263-2276.

Mok, D. W. and M. C. Mok. 2001. Cytokinin metabolism and action. Annu. Rev. Plant Physiol. Plant Mol. Biol. 52: 89-118.

Müller, B. 2011. Generic signal-specific responses: cytokinin and context-dependent cellular responses. J. Exp. Bot. 62: 32733288.

Niki, T., M. Hirai, T. Niki, A. Kanno and T. Nishijima. 2012. 
Role of floral homeotic genes in the morphology of forchlorfenuron-induced paracorollas in Torenia fournieri Lind. J. Japan. Soc. Hort. Sci. 81: 204-212.

Nishijima, T. 2007. Hanagata. p. 37-43. In Nosangyosonbunkakyokai (ed.). Nogyogijutsutaikei Kakihen 5 (Tsuiroku) 9 (In Japanese). Nosangyosonbunkakyokai, Tokyo.

Nishijima, T. and K. Shima. 2006. Change in flower morphology of Torenia fournieri Lind. induced by forchlorfenuron application. Sci. Hortic. 109: 254-261.

Nishijima, T., H. Miyaki, K. Sasaki and T. Okazawa. 2006. Cultivar and anatomical analysis of corolla enlargement of petunia (Petunia hybrida Vilm.) by cytokinin application. Sci. Hortic. 111: 49-55.

Nishijima, T., T. Niki and T. Niki. 2011a. Corolla of the largeflowered petunia (Petunia hybrida Vilm.) cultivars exhibit low endogenous cytokinin concentration through enhanced expression of the genes encoding cytokinin oxidases. J. Japan. Soc. Hort. Sci. 80: 334-342.

Nishijima, T., T. Niki and T. Niki. 2011b. The large-flowered petunia (Petunia hybrida Vilm.) genotype promotes expressions of type-A response regulator and cytokinin receptor genes like cytokinin response. J. Japan. Soc. Hort. Sci. 80: 343-350.

Pernisová, M., P. Klíma, J. Horák, M. Válková, J. Malbeck, P. Soucek, P. Reichman, K. Hoyerová, J. Dubová, J. Friml, E. Zažímalová and J. Hejátko. 2009. Cytokinins modulate auxininduced organogenesis in plants via regulation of the auxin efflux. Proc. Natl. Acad. Sci. USA 106: 3609-3614.
Reinhardt, D., T. Mandel and C. Kuhlemeier. 2000. Auxin regulates the initiation and radial position of plant lateral organs. Plant Cell 12: 507-518.

Rupp, H. M., M. Frank, T. Werner, M. Strnad and T. Schmülling. 1999. Increased steady state mRNA levels of the STM and KNATI homeobox genes in cytokinin overproducing Arabidopsis thaliana indicate a role for cytokinins in the shoot apical meristem. Plant J. 18: 557-563.

Schmülling, T., T. Werner, M. Riefler, E. Krupková and I. B. Manus. 2003. Structure and function of cytokinin oxidase/ dehydrogenase genes of maize, rice, Arabidopsis and other species. J. Plant Res. 116: 241-252.

Taniguchi, M., T. Kiba, H. Sakakibara, C. Ueguchi, T. Mizuno and T. Sugiyama. 1998. Expression of Arabidopsis response regulator homologs is induced by cytokinins and nitrate. FEBS Lett. 429: 259-262.

Troll, W. 1957. Praktische Einführung in die Pflanzenmorphologie 2. Fischer, Jena. (Troll, W. 2004. Zusetu-shokubutsukeitaigakuhandobukku 2 (In Japanese). Translated by S. Nakamura and H. Tobe. Asakurashoten, Tokyo.)

Venglat, S. P. and V. K. Sawhney. 1996. Benzylaminopurine induces phenocopies of floral meristem and organ identity mutants in wild-type Arabidopsis plants. Planta 198: 480-487.

Yamaguchi, H., T. Niki, T. Niki and T. Nishijima. 2010. Morphological property and role of homeotic genes in paracorolla development of Antirrhinum majus. J. Japan. Soc. Hort. Sci. 79: 192-199. 